\title{
ALLOZYME, SI GENE, CYTOLOGICAL, AND MORPHOLOGICAL POLYMORPHISMS IN A POPULATION OF OENOTHERA BIENNIS
}

\author{
Erich Steiner and Donald A. Levin \\ Dept. of Botany and Matthaei Botanical Gardens, University of Michigan, \\ Ann Arbor, Michigan, 48104 \\ and \\ Dept. of Botany, University of Texas, Austin, Texas, 78712 \\ Received October 27, 1975 \\ Revised May 25, 1976
}

The studies of population structure in the North American oenotheras by Cleland and his associates led to the conclusion that "over most of the North American range of the subgenus Oenothera, i.e. the area from the Rocky Mountains eastward, the population consists of innumerable true-breeding and therefore permanent complex-heterozygotes, each isolated from the others by reason of its self-pollinating habit but occasionally or rarely outcrossing, in which case hybrids are formed which tend to disappear if they have other than 014 , but which may become the progenitors of new permanent and isolated complex-heterozygous lines if they have 014." (Cleland, 1972, p. 230-231). The population of a given geographical region is composed of local stands which, in the main, appear to be discontinuous and modest in size, generally on the order of a few hundred individuals. Cleland's studies were based on collections which represented the major geographical regions of North America but only included a very limited, fortuitous sampling of particular local stands or unit populations. Population samples were characterized according to the segmental arrangements of the component chromosomal complexes and the phenotype which each complex produced. In the few cases where more than one member of a single stand was analyzed, variations in segmental arrangement were found, suggesting that local stands are heterogeneous, i.e. also composed of numerous, essentially isolated, inbreeding complex heterozygous lines. A precise knowledge of the genetic variability within the local stand can shed light on questions such as the nature of the colonization-extinction cycle, the significance of hybridization, and the degree of phenotypic plasticity exhibited by the inbred lines of complex-heterozygotes. Thus a method is needed which will allow rapid screening of adequate samples of local stands and will detect all inbred lines composing a stand. The determination of the segmental arrangement of a single collection requires on the average the production of 10-15 hybrids and their cytological analysis, an amount not feasible for large numbers of plants. Inbred lines in local stands of the biennis group 1 assemblage of Oenothera biennis have also been identified by analyzing collections for incompatibility alleles (Steiner, 1964; Al Khafaji and Steiner, 1970). This method for obtaining an index of genetic variation requires production of hybrids and extensive compatibility tests which limit the number of plants which can be surveyed during a season. The use of starch gel electrophoresis for identification of allozymes has shown particular promise as a method for rapidly surveying large samples of local stands for genetic variability (Levin et al., 1972; Levin, 1975). The allozyme studies as well as the results of incompatibility allele surveys supported the conclusion that single populations tend to be limited in variability, consisting of only one, or at the most, a few 
inbred lines. This finding contradicted the earlier, fragmentary cytological evidence on the variability of single populations by Cleland. The present study was undertaken to make a more detailed survey of genetic variability of a single population utilizing all three methods described above and to determine the extent to which the variability detected by each method could be correlated.

\section{Materials AND Methods}

Seeds were collected from each of 50 plants of a population of Oenothera biennis growing along railroad tracks on the southwestern outskirts of Roanoke, Virginia. The population, although composed to some extent of clusters, extended along the tracks for at least some 700 to 800 yards and into an adjacent vacant lot. The collections were made in sequence and at approximately equal intervals over the entire site. The collection numbers thus indicate the approximate location of the plant within the site.

Five plants were grown from each collection and established in the experimental garden; a sample of each seed collection was retained for allozyme analysis. The 50 cultures were studied for morphological variation. At least one plant in each culture was crossed as the seed parent to the synthesized complex-heterozygote, alpha Hot Springs - beta Camp Peary $L^{1}$. When used as a pollen parent, this hybrid transmits the alpha Hot Springs complex almost exclusively to the offspring, thus making it possible to obtain the alpha complex of each collection in a hybrid with alpha $H o t$

\footnotetext{
${ }^{1}$ According to the convention established by Cleland, a collection is given the name of the municipality where it is collected. Since most are complex-heterozygotes with two genomes remaining intact from generation to generation, the complexes are distinguished as alpha and beta. The alpha is defined as that complex which tends to be transmitted predominantly through the egg, the beta as the complex transmitted primarily through the pollen. The transmission behavior is variable to some extent, however, and depends upon the particular cross.
}

Springs. Such alpha $\cdot$ alpha hybrids are selfincompatible and can readily be analyzed for identity of incompatibility alleles by interpollination and determining the presence or absence of pollen tube growth in the styles. Further details on the method of incompatibility analysis can be found in Steiner (1964).

Chromosome configurations at meiosis of the alpha-alpha hybrids were determined utilizing smears of pollen mother cells prepared according to the method of Cleland (Cleland, 1972, Appendix II). For allozyme analysis individual two week old seedlings of each collection were assayed electrophoretically for the following enzyme loci: glutamate oxalate transaminase $(G o t)$, phosphoglucose isomerase (Pgi-1 and $P g i-2$ ) and esterase (Est) using procedures described by Levy and Levin (1975). Multiple molecular forms of these enzymes were known to occur in Oenothera biennis.

\section{Results}

Table 1 summarizes the results of the study. Complete or partial data were obtained for 28 of the original collections. Not all hybrids were grown; germination failures and inviability of certain hybrids also reduced the number of collections which could be analyzed.

Five different incompatibility alleles were identified among the alpha complexes of the collections studied. The different incompatibility alleles occur in general throughout the entire site and are not localized in particular segments of the collection site. Among the plants of each compatibility type, two different cytological configurations occur. Thus, alpha complexes which possess identical incompatibility alleles may, nevertheless, have different segmental arrangements; in no case, however, is the difference greater than one interchange. Alpha complexes among the incompatibility types $B, D$, and $E$ exhibit the typical alpha biennis group 1 segmental arrangement, namely $1 \cdot 2 \quad 3 \cdot 4 \quad 5 \cdot 14 \quad 7 \cdot 10$ $9 \cdot 811 \cdot 12 \quad 13 \cdot 6$. 
TABLE 1. Characteristics of Oenothera biennis from the Roanoke population.

\begin{tabular}{|c|c|c|c|c|c|c|}
\hline \multirow[b]{2}{*}{ Plant \# } & \multirow{2}{*}{$\begin{array}{c}\text { Incompatibility } \\
\text { class }\end{array}$} & \multirow{2}{*}{$\begin{array}{l}\text { Cytological } \\
\text { config } \\
\text { of hybrid }\end{array}$} & \multicolumn{4}{|c|}{ Allozyme Genotype of original collection } \\
\hline & & & Got & $P g i-1$ & $P g i-2$ & Est \\
\hline 2 & $A$ & $06,04,2 \mathrm{prs}$ & $b b$ & $b b$ & $b b$ & - \\
\hline 3 & $A$ & $06,04,2$ prs & $b b$ & $b b$ & $b b$ & $a a$ \\
\hline 4 & $A$ & $04,04,3$ prs & $b b$ & $b b$ & $b b$ & $a a$ \\
\hline 5 & $A$ & $04,04,3$ prs & $b b$ & $b b$ & $b b$ & $a a$ \\
\hline 6 & $A$ & $06,04,2 \mathrm{prs}$ & $b b$ & $b b$ & $b b$ & $a a$ \\
\hline 8 & $A$ & $06,04,2$ prs & $b b$ & $b b$ & $b b$ & $a a$ \\
\hline 13 & $A$ & $06,04,2$ prs & $b b$ & $b b$ & $b b$ & $a a$ \\
\hline 15 & $B$ & $7 \mathrm{prs}$ & $a a$ & $b b$ & - & $a a$ \\
\hline 16 & $B$ & $7 \mathrm{prs}$ & $a a$ & $b b$ & $b b$ & $a a$ \\
\hline 17 & $B$ & $7 \mathrm{prs}$ & $a a$ & $b b$ & $b b$ & $a a$ \\
\hline 18 & $B$ & - & $a a$ & $b b$ & $b b$ & $a a$ \\
\hline 19 & $C$ & $04,04,3 \mathrm{prs}$ & \multicolumn{4}{|c|}{ no runs } \\
\hline 20 & $C$ & $04,04,3$ prs & - & $b b$ & $b b$ & $a a$ \\
\hline 24 & $D$ & $7 \mathrm{prs}$ & $b b$ & $b b$ & $b b$ & $a a$ \\
\hline 25 & $E$ & $7 \mathrm{prs}$ & \multicolumn{4}{|c|}{ no runs } \\
\hline 29 & $D$ & 04,5 prs & $a b$ & $b b$ & $b b$ & $a b$ \\
\hline 31 & $A$ & $04,04,3 \mathrm{prs}$ & $b b$ & $b c$ & $b b$ & $a b$ \\
\hline 41 & $B$ & 04,5 prs & $a a$ & - & - & - \\
\hline 42 & $E$ & 04,5 prs & \multicolumn{4}{|c|}{ no runs } \\
\hline 44 & $B$ & - & $a a$ & - & - & - \\
\hline 46 & $B$ & 7 prs & $a a$ & $b b$ & $b b$ & $a a$ \\
\hline 47 & $B$ & $7 \mathrm{prs}$ & $a a$ & $b b$ & $b b$ & $a a$ \\
\hline 49 & $A$ & $04,04,3$ prs & $b b$ & $b b$ & $b b$ & $a a$ \\
\hline 50 & $C$ & $06,04,2$ prs & $b b$ & $b b$ & $b b$ & $a a$ \\
\hline \multicolumn{7}{|c|}{$\begin{array}{l}\text { Other allozyme genotypes occurring in population } \\
\text { for which incompatibility and cytological } \\
\text { data are not available }\end{array}$} \\
\hline 0 & & & $a a$ & $a a$ & $b b$ & $a b$ \\
\hline 1 & & & $a a$ & $b b$ & $b b$ & $a b$ \\
\hline 7 & & & $b b$ & $a a$ & $a a$ & $a a$ \\
\hline 36 & & & $a a$ & - & - & $a b$ \\
\hline
\end{tabular}

The allozyme genotypes parallel the incompatibility types closely. With one exception all plants of incompatibility type $A$ have the same allozyme genotype. Among those of incompatibility type $B$, the genotypes of three plants are incompletely determined, but those loci which are known do not contradict the assumption that only a single genotype is involved. For type $C$ the partial data still allow the assumption of a single allozyme genotype. Each of the two plants of incompatibility type $D$ has a distinctive allozyme genotype. The allozyme genotypes for type $E$ remain undetermined. Differences in incompatibility type are, however, not necessarily reflected in variation in allozyme genotype. For example, incompatibility types $A, C$, and $D$ all possess the same allozyme genotype, namely, Got ${ }^{b b}$ Pgi-1 $^{b b}$ Pgi-2 $^{b b}$ Est $^{a a}$.

Somewhat surprising is the relatively low frequency of heterozygosity at the allozyme loci. For Got one out of 24 determinations proved to be heterozygous; one genotype of 22 is heterozygous for Pgi-1,0 out of 21 for $P g i-2$, and 5 out of 22 for Est. Of the allozyme loci here considered the total heterozygosity is 0.078 . Only in one instance are there more than two alleles at a locus.

The original seed collections yielded cultures each of which was morphologically 
uniform with the exception of the culture arising from plant \#25; this culture consisted of two phenotypes. Both of these were selfed; at harvest time it was discovered that one of the types had failed to set seed. The following season a culture was grown from the phenotype which had been successfully selfed. This culture, like the previous generation, consisted of two phenotypes; further, one of them again failed to set seed and proved to be unquestionably self-incompatible. The selfcompatible strain appears to be segregating a self-incompatible line each generation. An analysis of this curious behavior has been undertaken.

While plants arising from each seed collection were morphologically identical with the exception of the culture described above, differences were readily apparent between many of the cultures. These differences involved largely quantitative characters such as degree of pigmentation, bud thickness and length, and extent of mildew susceptibility. Distinct morphological types can in general be correlated with the incompatibility types. For example, all of the plants of incompatibility type $A$ have the same phenotype with the exception of plants 13 and 49. Plant 13 has clear green instead of red stem tips, green and not red stem papillae and is mildew resistant in contrast to others of type $A$ which are mildew susceptible. Plant 49 is like other members of group $A$ but is mildew resistant.

All members of incompatibility group $B$ have the same appearance; they are characterized by white midribs, weakly red stem papillae, green stem tips, twisted and flared bracts, yellow, short, moderately slender buds, and a slight mildew susceptibility.

Those of type $C$ are similar to plant \#13 in morphology, but exhibit red papillae and stem tips. Plants of type $E$ are also like \#13 but strongly mildew susceptible. Plants of type $D$ are essentially like those of type $B$.

It should be noted that the variations in segmental arrangement of the alpha complexes of these collections do not correlate with their morphological variation.

As Table 1 shows, the alpha complexes of the Roanoke population mostly possess segmental arrangements which differ from one to three interchanges from that of alpha Hot Springs which is the typical arrangement of the biennis group I alpha complexes; seven plants, however, have alpha complexes with the typical arrangement. It should be noted that of the alpha-alpha hybrids which exhibited the configuration of seven pairs, i.e. were complete structural homozygotes, only one (derived from plant 24) matched the vigor of the alpha-alphas which exhibited one or more circles at meiosis. With the one exception, losses among those alpha-alphas with a configuration of seven pairs were high, often only one or two plants surviving in the field. In contrast, the alpha-alphas which were partial translocation heterozygotes equalled the original collections in vigor.

\section{Discussion}

Previous surveys of Oenothera biennis (Steiner, 1964; Al Khafaji and Steiner, 1970; Levin et al., 1972; Levin, 1975) led to the conclusion that local populations are made up of one, or at the most, a few genotypes. The present study suggests that this conclusion must be modified to recognize that at least some local populations are composed of an appreciable diversity of genotypes.

Why are some populations genetically depauperate and others diverse? Differences in the extent of variability within Oenothera biennis populations may depend upon the (1) number of genotypes among the colonizers, (2) age of the population, and (3) heterogeneity of the habitat.

Oenothera characteristically occurs in disturbed habitats. A single colonizer in such a habitat can give rise to a sizeable and genetically uniform population in the following season; however, if succession is rapid and a dense vegetational cover de- 
velops quickly, establishment of Oenothera seedlings in the following generation may become impossible so that the population cannot persist. On the other hand, on sites which remain continuously disturbed, colonization may occur repeatedly and involve genetically diverse colonizers. The succeeding populations will then be genetically variable. While the complex-heterozygotes of Oenothera are predominantly inbreeding, hybridization does occur, generating new inbred lines which add to the diversity. Finally, occurrence of mutations is well known in Oenothera; some of these may be preserved in the population and further augment the genetic variability. Thus, if the colonization-extinction cycle is of indefinite duration, variability can be expected to increase with the age of the population.

Moreover, if the disturbed area is environmentally heterogeneous, genetic polymorphism is likely to increase and be maintained through selection. Accordingly, we would expect relatively uniform populations in transient habitats (empty lots in cities and recent construction sites), but populations with greater variability in long occupied, more extensive and varied sites (e.g. railroad rights of way, the maintenance of which provides a continuously disturbed habitat).

Our findings are in accord with these expectations. Among the several Oenothera populations studied, the Roanoke population has the highest level of variability. The Roanoke population covered a larger area than the others and also included greater habitat variation. Populations from 44 localities in Illinois have less variability than the Roanoke population (Levin, 1975). For the most part, the former were growing on sites of recent disturbance and were small in standing crop relative to the Roanoke population. The populations in the Chicago region are growing on sites which have been suitable for Oenothera less than 25 years. The sites in downstate Illinois are much older. Differences in habitat heterogeneity among the Chicago and downstate sites were not obvious. Nevertheless, the downstate populations were the most variable of the Illinois sample. We have studied one population from Connecticut (Levin et al., 1972); almost all of the plants shared a common genotype. The population occupied a recently disturbed site.

The amount of heterozygosity at the allozyme loci is surprisingly low in the Roanoke population. Out of 28 plants 20 are homozygous at all loci tested. Thus it seems likely that the loci examined are not necessarily representative of the total genomes of the two complexes which make up these translocation heterozygotes. Further evidence for this view comes from observations of the vigor of the alpha-alpha hybrids produced for the incompatibility allele analysis. The striking increase in vigor that the alpha-alpha hybrids which are heterozygous for one to three interchanges show over those which are complete structural homozygotes cannot reasonably be attributed simply to the shift in chromosomal segments, i.e. the difference in vigor must result from a considerable genic heterozygosity on the chromosomes involved in the interchanges. Thus, a logical conclusion is that the Roanoke collections themselves, being complete translocation heterozygotes, must derive their vigor from a genic heterozygosity at least as great or greater than that of the partially segmentally heterozygous alpha-alpha hybrids. This heterozygosity, however, is not reflected in the allozyme results. In short, the complex heterozygotes must contain genic heterozygosity which remains undetected by present allozyme analyses.

The disparity between the presence of heterozygosity as revealed morphologically versus electrophoretically ostensibly is due to potential sources of bias inherent in gel electrophoresis as currently practiced (Lewontin and Hubby, 1966; Ayala et al., 1970). First, not all allelic variation is detectable by electrophoresis; thus the amount of heterozygosity within and among single organisms is probably underestimated, as is the level of polymorphism 
as a whole. Another important problem is that only one kind of gene, namely structural genes coding for soluble proteins and enzymes, can be studied. Perhaps Oenothera biennis and other permanent structural heterozygotes are highly heterozygous at regulatory loci. Indeed, the biochemical versatility which heterozygosity provides might be most highly elaborated at regulatory loci and expressed in terms of physiological luxuriance.

The considerable diversity of incompatibility alleles in the Roanoke population is puzzling. The studies of Lewis (1951) on mutation of the incompatibility locus in Oenothera organensis failed to demonstrate a single instance of mutation from one allelic form to another. For this reason it seemed more acceptable to view the different incompatibility alleles in populations of complex heterozygotes as relics derived from their structurally homozygous selfincompatible progenitor population in which there must have been strong selective pressure for $S I$ gene diversity (Steiner, 1964; Al Khafaji and Steiner, 1970). If, however, as our results show, a number of different alleles exist in even relatively small populations of complex heterozygotes, this view loses credibility, since it requires the assumption of repeated and massive hybridization between the structurally homozygous progenitors to give rise to the complex heterozygous populations. We are thus left with the alternative hypothesis which explains the diversity of incompatibility alleles as the result of continuing mutation of the locus in the complex heterozygotes. Whether a population of complex heterozygotes carries a single or a number of different incompatibility alleles is not relevant to the function of the allele as a pollen lethal in self-pollinations. Unless the locus affects a character in addition to compatibility behavior, mutations to a variety of allelic forms would appear to be selectively neutral and persist in the particular inbred lines in which they arose.

Our results indicate that no single form of analysis reveals all of the genetically distinct lines in the Roanoke population. At least seven lines were detected with gel electrophoresis, five lines on the basis of incompatibility types and four different chromosomal arrangements were identified. An analysis of allozyme loci not considered here might reveal additional polymorphism, as might new techniques for detecting protein polymorphism not manifested by charge differences on gels (cf. Bernstein et al., 1973; Singh et al., 1974). These advances notwithstanding, electrophoresis may still have limitations in describing genetic diversity in natural populations; variation at loci affecting other characters such as compatibility behavior, morphology, and physiological responses should not be overlooked.

\section{Summary}

A local stand of Oenothera biennis growing in Roanoke, Virginia was analyzed for variation in incompatibility alleles, chromosomal end arrangement, morphology, and selected allozyme loci. Among 28 plants for which data were obtained, 14 distinct inbred lines were identified. This significantly exceeded the number expected on the basis of earlier studies with single populations of Oenothera. Each method for surveying variability revealed some differences which went undetected by the other methods used. Evidence that the allozyme loci do not necessarily reflect all of the genic heterozygosity existing in a complex heterozygote is presented. Variability in local stands may depend upon the diversity of colonizers, the age of the population, i.e. the length of the colonization-extinction cycle, as well as the heterogeneity of the habitat.

\section{Literature Cited}

At Khafajt, S. D., and E. Steiner. 1970. Further analysis of Oenothera biennis populations for incompatibility alleles. Amer. J. Bot. 57: 183-189.

Ayala, F. J., C. A. Murao, S. Perez-Salas, R. Richmond, ANd T. Dobzhansky. 1970. Enzyme variability within the Drosophila willistoni group. I. Genetic differentiation among 
sibling species. Proc. Nat. Acad. Sci. 67:225232.

Bernstein, S. C., L. H. Throckmorton, AND J. L. Huвzy. 1973. Still more genetic variability in natural populations. Proc. Nat. Acad. Sci. 70:3928-3931.

Cleland, R. E. 1972. Oenothera:Cytogenetics and Evolution. Academic Press, New York.

Levin, D. A. 1975. Genic heterozygosity and protein polymorphism among local populations of Oenothera biennis. Genetics 79:477-491.

Levin, D. A., G. P. Howland, and E. Steiner. 1972. Protein polymorphism and genic heterozygosity in a population of the permanent translocation heterozygote, Oenothera biennis. Proc. Nat. Acad. Sci. 69:1475-1477.

Levy, M., ANd D. A. Levin. 1975. Genic heterozygosity and variation in permanent trans- location heterozygotes of the Oenothera biennis complex. Genetics 79:493-512.

LEwIs, D. 1951. Structure of the incompatibility allele. III. Types of spontaneous and induced mutation. Heredity 5:399-414.

Lewontin, R. C., AND J. L. Hubby. 1966. A molecular approach to the study of genic heterozygosity in natural populations. II. Amounts of variation and degree of heterozygosity in natural populations of Drosophila pseudoobscura. Genetics 54:595-609.

Singh, R. S., J. L. Hubby, and R. C. Lewontin. 1974. Molecular heterosis for heat sensitive enzyme alleles. Proc. Nat. Acad. Sci. 71: 1808-1810.

Sterner, E. 1964. Incompatibility studies in Oenothera: The distribution of $\mathrm{S}_{\mathrm{I}}$ alleles in biennis I populations. Evolution 18:370-378.

\section{ANNUAL MEETING OF THE AMERICAN SOCIETY OF ZOOLOGISTS AND SOCIETY OF SYSTEMATIC ZOOLOGY HOSTED BY THE CANADIAN SOCIETY OF ZOOLOGISTS}

The annual meeting of the American Society of Zoologists and the Society of Systematic Zoology hosted by the Canadian Society of Zoologists will be held December 27-30, 1977 in Toronto, Canada at the Royal York Hotel.

The following symposia are planned: Comparative Heart Physiology, Developmental and Evolutionary Aspects of Brain Endocrine Function, Antigen Recognition, Comparative Tumor Immunology, Comparative Auto-Immune Disease Models, Vertebrate Appendage Regeneration, Polyamines in Embryonic Development, Neuro- muscular Interactions, Marine Larvae: Metamorphosis and Settlement, Nectar Feeding in Birds, Adaptations to Adverse Environments, Physical Events that Affect Biotic Patterns, Evolutionary Mechanisms and Processes, and Phenetic and Phylogenetic Concepts and Methods of Classification.

A call for contributed papers will be issued in April 1977. For more details and forms write to: Ms. Mary Wiley, American Society of Zoologists, Box 2739 California Lutheran College, Thousand Oaks, Ca. 91360. 